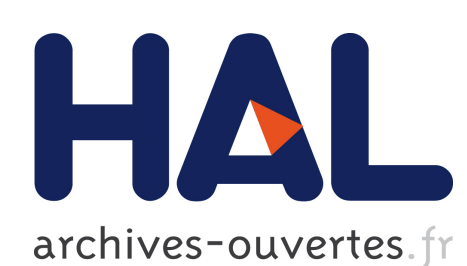

\title{
Problèmes de la théorie électronique du magnétisme
}

\author{
H.-J. Van Leeuwen
}

\section{To cite this version:}

H.-J. Van Leeuwen. Problèmes de la théorie électronique du magnétisme. J. Phys. Radium, 1921, 2 (12), pp.361-377. <10.1051/jphysrad:01921002012036100> . <jpa-00204299>

\section{HAL Id: jpa-00204299 \\ https://hal.archives-ouvertes.fr/jpa-00204299}

Submitted on 1 Jan 1921

HAL is a multi-disciplinary open access archive for the deposit and dissemination of scientific research documents, whether they are published or not. The documents may come from teaching and research institutions in France or abroad, or from public or private research centers.
L'archive ouverte pluridisciplinaire HAL, est destinée au dépôt et à la diffusion de documents scientifiques de niveau recherche, publiés ou non, émanant des établissements d'enseignement et de recherche français ou étrangers, des laboratoires publics ou privés. 


\section{LE JOURNAL DE PHYSIQUE \\ E T}

\section{LE RADIUM}

\section{PROBLĖMES DE LA THÉORIE ÉLEGTRONIQUE DU MAGNETISME}

Par Mlle H.-J. van LEEUIVEN.

\section{I. - Introduction.}

Dans cet article $\left(^{1}\right)$ la théorie électronique du magnétisme sera étudiée sur les cas les plus simples. Il y a encore de grandes divergences d'opinion sur les points principaux de cette théorie; il suffit, pour s'en rendre compte, de comparer les résultats de Langevin [13] (") et Kroo [12]. Le premier conclut, qu'on peut expliquer aussi bien le dia-que le paramagnétisme; le dernier, que la théorie électronique ne peut expliquer que le diamagnétisme. Il semble donc justifié de tirer jusqu'au bout les conséquences d'une méthode généralement admise, en l'appliquant aux hypothèses les plus simples qu'on puisse faire sur la constitution des molécules, aîn d'obterir des résultats auxquels on comparera ceux des auteurs précédents. C'est la méthode du théorème $\mathbf{H}$ de Boltzmann [2] que nous emploierons. Cette méthode est seulement applicable au cas des gaz, où les molécules ne s'influencent mutuellement dans leurs mouvements qu'aux moments des chocs et aux températures assez élevées pour que les lois de la mécanique classique soient encore valables, et que les quanta ne jouent aucun ròle. Nous négligerons aussi toute radiation due aux mouvements des charges électriques, sans quoi aucun état stationnaire n'est possible. Les mouvements doivent donc ètre quasi-stationnaires. L'applicalion de la méthode ne réussit toutà fait que pour des hypothèses spéciales faites sur la constitution de la molécule et sur le caractère des chocs. Ces hypothèses sont choisies en accord avec les travaux antérieurs. Quelques remarques sur le problème général suivront le trailement des cas spéciaux. La littérature relative à ce sujet sera discutée très brièvement.

(') Extrait d'une thèse soutenue à l'Université de Leyde, où se trouvent les démonstrations et les calculs supprimés ici. Elle est citée comme " thèse de Leyde ».

$(\stackrel{2}{)}$ Les nombres entre crochets se rapportent à la liste de référence placée à la fin de l'article.

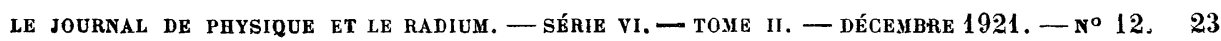




\section{II. - Définition des cas spéciaux.}

Trois cas de molécules, neutres quant-aux charges électriques totales qu'elles portent, seront traités :

$1^{\circ}$ Molécules portant un courant, e'est-à-dire molécules qui sont ellesmèmes des ellipsoïdes de révolution portant dans l'équateur une orbite où l'électricité positive est fixe, tandis que l'électricité négative peut y circuler librement.

$2^{\circ}$ Molécules portant des charges, c'est-à-dire molécules ayant à l'intérieur de la surface d’un ellipsoïde de révolution une densité constante de masse et de charge. Pour neutraliser la molécule nous pouvons nous imaginer une égale quantité de charges de signe opposé, situées dans une petite sphère au centre; si cette sphère a une masse et des moments d'inertie beaucoup plus grands que l'ellipsoïde, nous en pouvons négliger l'effet magnétique, parce que ce dernier est d'autant plus petit que le quotient de la charge par la masse ou le quotient de la charge par le moment d'inertie sont plus petits.

$3^{\circ}$ Doublets ou molécules consistant en deux particules sphériques ayant des charges égales et opposées et s'attirant avec une force fonction arbitraire de leur distance mutuelle.

Pour chacun des cas nous introduirons deux hypothèses différentes sur les chocs.

Enfin l'effet magnétique des électrons libres des métaux sera étudié.

\section{III. - Le Théorème de Boltzmann.}

Généralement, l'état de la $x^{\text {me }}$ molécule sera fixé par $n$ coordonnées $q_{1}{ }^{(x)}, \ldots q_{v}{ }^{(x)}, \ldots q_{n}{ }^{(x)}$ et autant de composantes de vitesse : $\dot{q}_{1}{ }^{(x)}, \ldots \dot{q}_{v}{ }^{(x)}, \ldots \dot{q}_{n}{ }^{(x)}$. Soit $L$ la fonction de Lagrange (1) du système étudié; les équations du mouvement s'écrivent sous la forme :

$$
\frac{\mathrm{d}}{\mathrm{d} t}\left(\frac{\partial L}{\partial \dot{q}_{\nu}}\right)-\frac{\partial L}{\partial q_{\nu}}=Q_{v}\left({ }^{2}\right)
$$

où $Q_{\nu}$ représente la composante d'ordre v de la résultante des forces exercées sur la molécule. Nous pouvons introduire, au lieu des $\dot{q}_{\nu}(\nu=1, \ldots n)$, les moments $p_{v}$ définis par

$$
p_{\mathrm{v}}=\frac{\partial L}{\partial \dot{q}_{v}}
$$

(i) $L$ est Ia différence entre les énergies cinétique et potentielle, éventuellement augmentée de la différence des énergies magnétique et électrique.

$\left({ }^{2}\right)$ Les indices $(x)$ ne seront donnés que Iorsque la clarté l'exigera. 
Nous cherchons maintenant quel est le nombre $f\left(q_{1} \ldots q_{n} \cdot p_{1} \ldots p_{n}\right) \mathrm{d} \omega$ de molécules “ situées » (') dans un élément $\mathrm{d} \omega$ de l'extension en phase, à $2 n$ dimensions. dans laquelle les $q$ et les $p$ sont les coordonnées.

Pour chacun des cas que nous étudierons, nous devrons démontrer le théorème de Liouville, c'est-à-dire qu'un groupe de molécules $f \mathrm{~d} \omega$ reste toujours, pendant son mouvement, dans des éléments d'extension en phase, égaux à $\mathrm{d} \omega$, tandis que dans le cas du choc de deux molécules ce même résultat est valable dans une extension en phase où les coordonnées et moments de ces deux molécules figurent comme coørdonnées d'un mème point de l'extension. Boltzmann a démontré que, ce théorème étant valable, si par un choc quelconque d'une molécule, qui se trouve dans l'état $\left(q_{1}^{(1)}, \ldots q_{n}^{(1)}, p_{1}^{(1)} \ldots p_{n}{ }^{(1)}\right)$ arec une molécule $\left(q_{1}^{(2)}, \ldots q_{n^{(2)}}\right.$, $\left.p_{1}{ }^{(2)} \ldots p_{n}{ }^{(2)}\right)$, leurs coordonnées et moments se transforment en $\left(q_{1}{ }^{(1)^{\prime}}, \ldots q_{n}{ }^{(1)^{\prime}}\right.$,

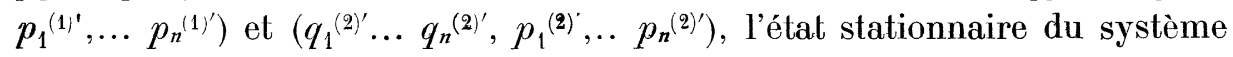
est caractérisé par la condition $\left({ }^{2}\right)$ :

$$
f\left(q_{1}^{(1)}, \ldots p_{n^{(1)}}^{(1)} f\left(q_{1}^{(2)}, \ldots p_{n^{(2)}}^{(2)}=f\left(q_{1}^{(1)^{\prime}}, \ldots p_{n^{(1)^{\prime}}}\right) f\left(q_{1}^{(2)^{\prime}}, \ldots p_{n}{ }^{(2)^{\prime}}\right),\right.\right.
$$

que nous écrirons en abrégé :

$$
f_{1} f_{2}=f^{\prime}{ }_{1} f_{2}
$$

Si l'équation (1) est satisfaite, chaque groupe de molécules gagne, pạr suite des collisions, autant de molécules qu’il en perd. Si les molécules du groupe $f \mathrm{~d} \omega$ n'étaient pas sujettes aux chocs, elles viendraient, après un temps $t$, en $d \omega_{1}$. En réalité, les mèmes molécules ne viennent pas après ce temps $t$ dans $\mathrm{d} \omega_{1}$, mais il y arrive autant de molécules qu'il y en avait avant $t$ dans $\mathrm{d} \omega$. Comme $\mathrm{d} \omega_{1}=\mathrm{d} \omega$, la densité est après l'instant $t$ la mème dans $\mathrm{d} \omega_{1}$ qu'arant cet instant dans $\mathrm{d} \omega$. Afin que l'état soit stationnaire, il faut donc que la mème densité en phase existe dans toutes les positions qui seraient occupées par une molécule si elle se mouvait sans chocs, ce qui s'exprime par l'équation :

$$
\frac{\mathrm{d} f}{\mathrm{~d} t}=\sum_{\nu=1}^{\nu=n} \frac{\partial f}{\partial q_{v}} \dot{q}_{\nu}+\sum_{\nu=1}^{\nu=n} \frac{\partial f}{\partial p_{v}} \dot{p}_{\nu}=0,
$$

laquelle s'applique comme s'il n'y avait point de chocs (c'est-à-dire avec $Q_{v}{ }^{\prime}=0$, si $Q_{v}{ }^{\prime}$ désigne une force de collision).

( $\left.{ }^{1}\right)$ Ceci étant une expression simplifiée qui exprime que le point de coordonnées $q_{1}, \ldots$. $q_{n}, p_{1}, \ldots p_{n}$, qui représente la position d'une molécule dans l'extension en phase, essitué dans $d \omega$.

(2) La démonstration de (1) se trouve dans la thèse de Leyde. 
La solution la plus générale des équations (1) et (2) sera déduite pour chacune des trois sortes de molécules (et aussi pour les électrons libres dans les métaux). Parfois il faudra encore se servir de relations de symétrie du système.

\section{IV. - Le théorème de Liouville.}

Pour la démonstration générale du théorème de Liouville, nous nous servirons des équations du mouvement sous la forme :

$$
\frac{\mathrm{d}}{\mathrm{d} t}\left(\frac{\partial L}{\partial \dot{q}_{\nu}}\right)-\frac{\partial L}{\partial q_{\nu}}=()_{\nu}+{()_{\nu}^{\prime}}_{\nu} \quad(\nu=1,2, \ldots u)
$$

(voir [14] p. 170).

Les $Q_{v}{ }^{\prime}$ seront les forces réciproques exercées par les molécules au moment d'une collision; quant-aux $Q_{\vee}$ et $L$ deux cas se présentent :

$1^{\circ}$ Les forces électromagnétiques sont comprises dans $L$, cette fonction élant la différence des énergies cinétique et potentielle, augmentée de celle des énergies magnétique et électrique; les $Q_{v}$ sont nuls ( $\left.{ }^{1}\right)$.

$2^{\circ} L$ est seulement la différence des énergies cinétique et potentielle, alors les $Q_{v}$ représentent les forces exércées par le champ magnétique.

Dans tous les cas nous introduirons les moments au moyen des équa. tions :

$$
p_{v}=\frac{\partial L}{\partial \dot{q}_{v}}
$$

et une fonction nouvelle $R$, que nous définirons par :

$$
R=\sum_{1}^{k} \sum_{1}^{n} \frac{\partial L}{\partial \dot{q}_{\nu}{ }^{(x)}} \dot{q}_{\nu}{ }^{(x)}-L
$$

Nous la nommons fonction de Routh, parce que la somme, dans (4), ne s'étend pas à toutes les coordonnées (pas à $q_{o}$ ) ( $\left.{ }^{1}\right)$.. Si la somme était étendue à toutes les coordonnées, nous aurions la fonction de Hamilton $R$ s'exprime en fonction des $p$ et $\operatorname{des} q$, et non pas en fonction des $\dot{q}$. Les $Q$, qui étaient fonctions des $q$ et des $\dot{q}$, s'expriment maintenant au moyen des $q$ et des $p$.

Les équations du mouvement se transforment alors en $\left(^{2}\right)$ :

(1) Le courant, producteur du champ magnétique, est alors compris dans le système. Nous désignerons par $q_{o}$ la coordonnée, qui mesure la position de l'électricité dans l'orbite de ce courant. e'est-à-dire la quantité d'électricité qui a traversé une certaine section donnée depuis un certain instant initial. $L$ ne dépend alors que de la vitesse $\dot{q}_{o}$, l'intensité du courant, et non pas de $q_{o}$, qui est une coordonnée cyclique.

(2) Voir thèse de Leyde. 


$$
\dot{q}_{v}=\frac{\partial R}{\partial p_{v}}, \quad \dot{j}_{v}=-\frac{\partial R}{\partial q_{v}}+Q_{v}+Q_{v}{ }^{\prime}
$$

Il faut démontrer maintenant le théorème de Liouville, sous la forme $\Delta=1$, si $\Delta$ est le déterminant suivant :

$$
د=\frac{\partial\left(q_{1}^{\prime}, \ldots q_{n}^{\prime}, p_{1}^{\prime}, \ldots p_{n}^{\prime}\right)}{\partial\left(q_{1}, \ldots q_{n}, p_{1}, \ldots p_{n}\right)}=\frac{\partial\left(q_{1}+\dot{q}_{1} \mathrm{~d} t, \ldots p_{n}+\dot{p}_{n} \mathrm{~d} \ell\right)}{\partial\left(q_{1}, \ldots p_{n}\right)} .
$$

Cette démonstration s'obtient, si on néglige les termes du second ordre en $\mathrm{d} t$, et si on applique les formules (5). Dans le second cas, on utilise le fait que les $Q$ ne produisent pas de travail dans le mouvement réel $\left({ }^{1}\right)$.

Pour le cas où il y a des collisions, il faut encore démontrer que

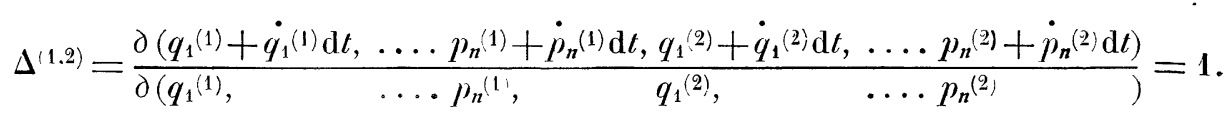

La preuve s'obtient comme la précédente, si on se sert encore de la relation $\frac{\partial Q_{v}^{\prime}}{\partial p_{v}}=0$, donc si on, suppose que les forces au moment du choc ne dépendent pas des vitesses.

\section{V. - Effet magnétique des molécules portant un courant.}

Dans la molécule ellipsoïdale, nous fixons un système d'axes de référence $O \Xi \mathrm{HZ} ; O \Xi$ est l'axe de rotation. Nous en définissons la position par rapport à un système $O X Y Z$, fixe dans l'espace et dont l'axe $O X$ soit parallèle aux lignes de force, au moyen des angles Eulériens $q_{1}, q_{2}, q_{3}$. Le premier, $q_{1}$, est l'angle entre $O X$ et $O \Xi ; q_{2}$, celui des plans $X O \Xi$ el $X O Y ; q_{3}$, celui des plans $X O \Xi$ et $\Xi O H$. Un méridien fixé au cercle d'électricité mobile fait l'angle $q_{l}$ avec un méridien fixé à la molécule. Le champ magnétique a l'intensité $H$ et le courant extérieur, qui produit ce champ $\boldsymbol{H}$, a la vitesse $\dot{q}_{o}$; la fonction de Lagrange s'écrit, si nous prenons pour ce cas la somme de l'énergie cinétique et magnétique (l'énergie potentielle des forces de choc n'étant pas comprise dans $L$ el l'énergie électrique étant nulle) :]

$$
\begin{aligned}
& L=\Sigma\left[\frac{m}{2}\left(\dot{q}_{x}{ }^{2}+\dot{q}_{y}{ }^{2}+\dot{q}_{z}\right)+\frac{\alpha}{2}\left(\dot{q}_{2} \cos q_{1}+\dot{q}_{3}\right)^{2}+\frac{\beta}{2}\left(\dot{q}_{1}{ }^{2}+\dot{q}_{2}{ }^{2} \sin ^{2} q_{1}\right)+\frac{\lambda}{2} \dot{q}_{l}{ }^{2}+\dot{q}_{l} \dot{q}_{0} \cos q_{1}\right] \\
& +\frac{y}{2} \dot{q}_{o}^{2}
\end{aligned}
$$

La somme s’étend à toutes les molécules, $\alpha$ et $\beta$ sont les moments

(1) Voir thèse de Leyde. 
d'inertie par rapport à l'axe $O \Xi$ et aux axes $O H$ el $O Z ; \gamma$ et $\lambda$ sont les coefficients de self-induction du courant moléculaire et du courant extérieur, $\mu$ leur coefficient d'induction mutuelle ${ }^{1}{ }^{1}$ si les circuits sont parallèles et les directions positives pour $q_{l}$ et $q_{0}$ les mèmes ; $q_{x}, q_{y}$, et $q_{z}$ sont les coordonnées du centre de gravité.

L'introduction d'une fonction de Routh et des moments $p$ est désirable pour des raisons diverses:

$1^{\circ}$ Le théorème de Liouville ne serait pas valable si nous prenions dans l'extension en phase les composantes de vitesse comme coordonnées au lieu des moments. Les équations du mołvement ont, pour les moments, la forme canonique :

$$
\dot{q}_{v}=\frac{\partial R}{\partial p_{v}}, \quad \dot{p}_{v}=-\frac{\partial R}{\partial q_{\nu}}+Q_{v}^{\prime}
$$

pour $q_{\mathfrak{c}}$, l'équation a, au contraire, la forme :

$$
\frac{\mathrm{d}}{\mathrm{d} t}\left(\frac{\partial R}{\partial \dot{q}_{o}}\right)-\frac{\partial R}{\partial q_{o}}=-Q_{o}
$$

$2^{\circ} R$ est constant, si nous supposons $\dot{q}_{\text {o (et aussi liintensité } \boldsymbol{i} / l}$ du champ magnétique) constant, ce que nous admeltrons pour obtenir la solution des équations (1) et (2). $\left({ }^{2}\right)$.

$3^{\circ} R$ est préférable à l'énergie totale, si nous les exprimons toutes deux au moyen des moments convenables. Si nous avions introduit aussi le moment $p_{o}=\frac{\partial L}{\partial \dot{q}_{o}}$, la transformation eùt été complète, de sorte que l'équation en $q_{o}$ eùt été canonique elle aussi. Alors $E$ serait resté constant (si on avait supposé le moment $p_{o}$ constant, et non $\dot{q}_{o}$ ni $H$ ), mais $E$ ne se serait pas écrite sous forme d'une somme de termes qui se rapportent chacun à une seule molécule, et d'une constante, ce qui est bien le cas pour $\boldsymbol{R}\left({ }^{3}\right)$. C'est une simplification importante pour le calcul avec $R$.

$$
\begin{aligned}
R=\sum\left[\frac { 1 } { 2 m } \left(p_{x}{ }^{2}+p_{y}{ }^{2}\right.\right. & \left.+p_{z}^{2}\right)+\frac{1}{2}\left\{\frac{1}{\beta} p_{1}^{2}+\frac{1}{\beta \sin ^{2} q_{1}}\left(p_{2}-p_{3} \cos q_{1}\right)^{2}+\frac{1}{\alpha} p_{3}{ }^{2}\right\} \\
& \left.+\frac{1}{2 \lambda}\left\{p_{l}{ }^{2}-2 \mu \dot{q}_{o} p_{l} \cos q_{1}+\dot{q}_{o}{ }^{2} \mu^{2} \cos ^{2} q_{1}\right\}\right]-\frac{\nu}{2} \dot{q}_{o}{ }^{2}
\end{aligned}
$$

(1) $\lambda$ et $\mu$ ne sont pas les coefficients d'induction au sens ordinaire, mais en diffèrent par un facteur constant, parce que $q_{l}$ est un angle et non pas une quantité d'électricité écoulée.

$\left(^{2}\right)$ Voir thèse de Leyde : ce n'est pas le cas pour $E=R-\frac{\partial R}{\partial \dot{q}_{0}} \dot{q}_{o}$, énergie totale.

$\left({ }^{3}\right)$ Voir thèse de Leyde. 
La première hypothèse sur le choc sera, que le mourement du circuit ne soit pas influencé par le choc entièrement élastique dës deux ellipsoïdes. Si nous posons $\log f=\varphi_{\text {, }}$ on peut remplacer (1) par *

$$
\varphi^{(1)}+\varphi^{(2)}=\varphi^{\prime 1)^{\prime}}+\varphi^{(2)^{\prime}}
$$

Il faut que cette équation se déduise des équations entre les variables qui fixent la position, dans l'extension en phase, de la molécule avant et après le choc. Si la durée du choc est infiniment petite, ces équations sont telles que les $q$ restent constants, les $p_{3}$ aussi (parce qu'il n'y a pas de moment sur les axes $O \Xi) ; p_{x}^{(1)}+p_{x}^{(2)}, p_{y}^{(1)}+p_{y}^{(2)}, p_{z}^{(1)}+p_{z}^{(2)}$, les $p_{t}, \boldsymbol{R}^{(1)}+\boldsymbol{R}^{(2)}$ restent invariables ainsi que quatre équations de moments. Ces quatre dernières équations sont les seules qui n'aient point la forme analogue à (7) :

$$
x^{(1)}+x^{(2)}=x^{(1)^{\prime}}+x^{(2)^{\prime}} .
$$

On voit immédiatement que l'on peut écrire

$$
\varphi=-\varepsilon_{o} R+m_{x} p_{x}+m_{y} p_{y}+m_{z} p_{z}+\psi\left(p_{3}, p_{l}, q_{1}, q_{2}, q_{3},\right),
$$

où $\varepsilon_{o}, m_{x}, m_{y}$ et $m_{z}$ sont des constantes et $\psi$. une fonction arbitraire qui satisfait à l'équation (7). La démonstration que c'en est la solution la plus générale $\left({ }^{1}\right)$ ne sera pas donnée ici. Si nous employons encore l'équation (2), nous trourons comme forme définitive

$$
v=-\varepsilon_{o} R+H\left(p_{3}, p_{l}\right)+m_{x} p_{x}+m_{y} p_{y}+m a_{z} p_{z} .
$$

Le moment magnétique dans la direction $O X$ de chaque molécule est : $\delta \dot{q}_{l} \cos q_{1}$ ( $\delta$ étant une constante); la valeur moyenne du moment de toutes les molécules sera :

$$
\bar{m}=\frac{\frac{\delta}{\lambda} \int\left(p_{t} \cos q_{1}-\mu \dot{q}_{o} \cos ^{2} q_{1}\right) \mathbf{e}^{-\varepsilon_{o} R+m_{x} p_{x}+m_{3} p_{y}+m_{z} p_{s}+H\left(p_{3}, p_{l}^{\prime}\right.} \mathrm{d} \omega}{\int \mathbf{e}^{-\varepsilon_{0} R+m_{x} p_{x}+m_{y} p_{y}+m_{z} p_{*}+H\left(p_{3}, p_{l}\right)} \mathrm{d} \omega} .
$$

On trouve donc pour le moment magnétique de l'unité de volume, si $N$ est le nombre de molécules contenues dans ce volume, et si l'on peut développer suivant les puissances de $H x$, où $x=\cos q_{1}$ :

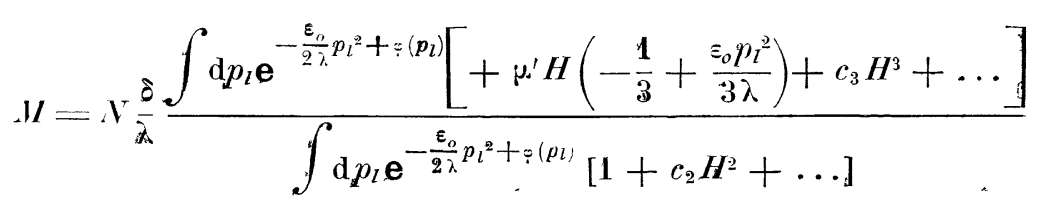

(') Voir thèse de Lueyde. 
où l'on peut exprimer les $c$ aisément au moỵen des $\varepsilon_{o}$, $\mu^{\prime}$ ' et $p_{l}$, si $\mu^{\prime}=\frac{\mu_{\dot{q}}}{H}$

Il y a donc diamagnétisme, si :

$$
\frac{1}{3} \int \mathrm{d} p_{l} \mathrm{e}^{\left.-\frac{\varepsilon_{o}}{2 \lambda} p_{l}{ }^{2}+\xi_{l}\right)}>\frac{\varepsilon_{o}}{3 \lambda} \int \mathrm{d} p_{l} \mathrm{e}^{-\frac{\varepsilon_{o}}{2 \lambda} p_{l}+\xi\left(p_{l}\right)} p_{l}^{2},
$$

et paramagnétisme si la seconde expression est plus grande que la première. Le cas le plus simple se présente s'il n'y a qu'une valeur de $p_{l}$, soit $p$, pour toutes les molécules. Il ne reste des intégrales qu'un seul terme :

$$
M=N \frac{\delta}{\lambda} \mu^{\prime} H\left(-\frac{1}{3}+\frac{\varepsilon_{o} p^{2}}{3 \lambda}\right)
$$

pour les pelites valeurs de $\boldsymbol{I}$. Nous concluons :

$1^{\circ}$ Si le moment $p$ est nul pour chaque molécule, le gaz est diamagnétique avec une susceplibilité indépendante de la température :

$$
-\frac{1}{3} N \frac{\delta}{\lambda} \mu^{\prime} ;
$$

$2^{\circ}$ Si le moment $p$ est, au contraire, très grand, le moment $M$ est approximativement $\varepsilon_{0} N_{3} \frac{\delta}{\lambda^{2}} \mu^{\prime} H p^{2}$.L'énergie cinétique moyenne des molécules pour le mouvement de translation est $\frac{3}{2 \varepsilon_{o}}$, on peut donc écrire $k 7$ au lieu de $\frac{1}{\varepsilon_{o}}$. Le moment paramagnétique varie donc en raison inverse de la température.

Si les collisions ne sont pas instantanées, mais si leur durée est courte par rapport à la durée du mouvement entre deux chocs et si nous tenons compte des forces magnétiques exercées mutuellement par les molécules un moment du choc, on peut encore démontrer que la distribution selon $(9)$ est stationnaire, mais il est beaucoup plus difficile de démontrer qu'elle est la seule distribution stationnaire possible.

Nous examinerons une deuxième hypothèse sur le caractère du choc. Dans le cas précédent, la fonction distributrice ne pouvait contenir que les combinaisons des variables $; \boldsymbol{R}, p_{\boldsymbol{x}}, p_{\boldsymbol{y}}, p_{z}, p_{3}$ et $p_{l}$. Le moment magnétique ne dépendait pas de $p_{x}, p_{y}, p_{z}$ et $p_{3}$, de sorte que la distribution arbitraire, quant à ces grandeurs, restait sans influence; c'était seulement celle relative à $p_{l}$, qui jouait un rôle important. C'est pour étudier ce que devient $\boldsymbol{M}$, si $p_{l}$ peut varier au moment des chocs, que nous introduirons une hypothèse 
plus artificielle sur le caractère des chocs. Supposons le gaz mélangé à un gaz monoatomique, dont les molécules peuvent cntrer en collision avec les premières dans un plan méridien $\Xi O \mathrm{H}_{1}$, lié au cercle mobile que portent celles-ci. Pour simplifier nous supposons encore que le choc ne peut se produire que contre une bande étroite de $\Xi O \mathrm{H}_{1}$, dans l'équateur de la molécule, de sorte que l'axe du couple résultant du choc coïncide avec l'axe de la molécule. Ce cas nous sert de transition avec celui où le mouvement de l'électricité dans la molécule consiste dans la circulation de particules chargées séparées. Comme dans le cas précédent, nous cherchons les relations entre les valeurs des variables avant et après le choc, et nous trouvons comme solution; $\varphi=-\varepsilon_{o} R+F$, où $F$ représente une fonction qui ne dépend pas de $p_{l}$. Le moment moléculaire est

$$
\dot{\delta} \dot{q}_{l} \cos q_{1}=\delta \frac{\partial R}{\partial p_{l}} \cos q_{1} .
$$

Si on intègre l'expression du moment résultant :

$$
M=\delta_{N} V \frac{\int \frac{\partial R}{\partial p_{l}} \cos q_{1} e^{-\xi_{0} R+F} \mathrm{~d} q_{1} \mathrm{~d} q_{2} \mathrm{~d} q_{3} \mathrm{~d} p_{1} \mathrm{~d} p_{2} \mathrm{~d} p_{3} \mathrm{~d} p_{l} \mathrm{~d} p_{x} \mathrm{~d} p_{r} \mathrm{~d} p_{z}}{\int \mathrm{e}^{-\varepsilon_{0} R+F} \mathrm{~d} q_{1} \mathrm{~d} q_{2} \mathrm{~d} q_{3} \mathrm{~d} p_{1} \mathrm{~d} p_{2} \mathrm{~d} p_{3} \mathrm{~d} p_{l} \mathrm{~d} p_{x} \mathrm{~d} p_{y} \mathrm{~d} p_{z}}
$$

par rapport à $p_{l}$, le résultat est zéro. Le moment magnétique est donc nuI dans ce cas, où le mouvement de l'électricité subit des perturbations par suite des chocs.

\section{VI. - Effet magnétique des molécules portant des charges.}

Nous introduisons comme fonction de Lagrange l'énergie cinétique seule; c'est, pour chaque molécule :

$$
T=\frac{m}{2}\left(\dot{q}_{x}{ }^{2}+\dot{q}_{y}{ }^{2}+\dot{q}_{z}{ }^{2}\right)+\frac{\alpha}{2}\left(\dot{q}_{2} \cos q_{1}+\dot{q}_{3}\right)^{2}+\frac{\beta}{2}\left(\dot{q}_{1}{ }^{2}+\dot{q}_{2}{ }^{2} \sin ^{2} q_{1}\right) .
$$

La fonction de Routh devient alors :

$$
R=\frac{1}{2 m}\left(p_{x}{ }^{2}+p_{y}{ }^{2}+p_{z}^{2}\right)+\frac{1}{2}\left[\frac{1}{\beta} p_{1}{ }^{2}+\frac{1}{\beta \sin ^{2} q_{1}}\left(p_{2}-p_{3} \cos q_{1}\right)^{2}+\cdot \frac{1}{\alpha} p_{3}{ }^{2}\right] .
$$

En plus des forces $Q_{v}{ }^{\prime}$, qui n'existent qu'au moment d'une collision, il y a maintenant des forces $Q_{v}$ exercées par le champ magnétique; mais les $Q_{v}$ ne produisent pas de travail, et l'on a pour chaque molécule, excepté aux moments des chocs :

$$
\boldsymbol{R}^{(\boldsymbol{x})}=\text { Constante; }
$$


au moment d'un choc pour les deux molécules (1) et (2),

$$
R^{(1)}+R^{(2)}=R^{(1)^{\prime}}+R^{(2)^{\prime}} \text {. }
$$

Comme première hypothèse sur le choc, supposons que les deux ellipsoïdes se choquent tout à fail élastiquement. On a, si on élimine directement $q_{x}, q_{y}$ et $q_{z}$, dont la fonction distributrice ne peut dépendre, 16 équations accessoires : les $q$ restent constants, les $p_{x}^{(1)}+p_{x}{ }^{(2)}, p_{y}{ }^{(1)}+p_{y}{ }^{(2)}$, $p_{z^{(1)}}+p_{z^{(2)}}$ restent constants, les $p_{3}$ restent constants, et enfin 4 équations pour les moments.

On démontre que la solution des équations (1) et (2), avec ces équations accessoires, est :

$$
\varphi=-\varepsilon_{0} R+H\left(p_{3}+\alpha h \cos q_{1}\right)+m_{x} p_{x}+m_{y} p_{y}+m_{z} p_{z},
$$

où $\varepsilon_{o}, m_{x}, m_{y}$ et $m_{z}$ sont des constantes et $h=\frac{e H}{2 \rho c}$, si $\rho$ est la densité de masse et $e$ la densité de l'électricité.

Le moment magnétique d'une molécule parallèle à $O X$ est $\frac{e}{2 \rho^{\circ}} p_{2}$; le moment de l'unité de volume, contenant $N$ molécules, s'écrit donc. après quelques simplifications, et par l'introduction de $p=p_{3}+\alpha h \cos q_{1}$ et $x=\cos q_{1}$ :

$$
M=\frac{N e}{2 \rho c} \frac{\iint_{-1}^{+1} \mathrm{e}^{-\frac{\varepsilon_{0}}{\alpha_{\alpha}}\left(p^{2}-2 \alpha h p x+\alpha^{2} h^{2} x^{2}\right)+H(p)}(p-\alpha h x) x \mathrm{~d} x \mathrm{~d} p}{\iint_{-1}^{+1} \mathrm{e}^{-\frac{\varepsilon_{0}}{2 \alpha}\left(p^{2}-2 \alpha h p x+\alpha^{2} h^{2} x^{2}\right)+H(p)} \mathrm{d} x \mathrm{~d} p}
$$

Si on développe suivant les puissances de $h x$ et qu'on intègre par rapport à $x$, cette formule prend la mème forme que la formule (10). On en peut tirer les mèmes conséquences. Dans ce cas, comme dans l'autre, il peut se passer que $\boldsymbol{I I}$ exclue toute valeur de $p$ exceptée une seule. Si cette valeur est grande par rapport au changement que les moments subissent de la part du champ magnétique, on a paramagnétisme en raison inverse de la température; si elle est nulle, on a diamagnétisme, indépendant de la température. En peu de mots, il sera évident que si des chocs d'autre caractère pouvaient se produire encore, où $R^{(1)}+R^{(2)}$ resterait constante, mais non pas $p=p_{3}+\alpha h \cos q_{1}$, une distribution régie par la fonction

$$
f=C \mathbf{e}^{-\varepsilon_{o} R+m_{x} p_{x i} i+m_{y} p_{y}+m_{z} p_{z}}
$$

serait seule stationnaire. Le moment résultant serait alors zéro, ce qui se déduit de (11) en introduisant $\boldsymbol{H}=0$ et en intégrant par rapport à $p$. 
VII. - Effet magnétique des doublets.

Nous distinguons les deux particules d'une molécule par les indices $\alpha$ et $\beta$; deux molécules en collision, par les indices (1) et (2); les états avant et après le choc, respectivement par des lettres non accentuées et accentuées. La première molécule se compose donc d'une particule sphérique $\alpha$ de charge $+e$, de coordonnées $x_{\alpha_{1}}, y_{\alpha_{1}}, z_{\alpha_{1}}$ et de composantes de vitesse $\dot{x}_{\alpha_{1}}, \dot{y}_{\alpha_{1}}, \dot{z}_{\alpha_{1}}$ et une particule sphérique $\beta$ de charge $-e$, de coordonnées $x_{\beta_{1}}, y_{\beta_{1}}, z_{\beta_{1}}$, et de composantes de vitesse $\dot{x}_{\beta_{1}}, \dot{y}_{\beta_{1}}, \dot{z}_{\beta_{1}}$; pour la deuxième molécule les notations sont analogues. Nous employons la différence des énergies cinétique et potentielle comme fonction de Lagrange. Dans ce cas les moments ne diffèrent des composantes de vitesse que par des facteurs constants; on peut donc employer aussi bien ces dernières comme coordonnées dans l'extension en phase. L'énergie cinétique est :

$$
T=\frac{m_{\sigma}}{2}\left(\dot{x}_{\alpha^{2}}{ }^{2}+\dot{y}_{\alpha^{2}}{ }^{2}+\dot{z}_{\alpha_{2}}{ }^{2}\right)+\frac{m_{3}}{2}\left(\dot{x}_{9}{ }^{2}+\dot{y}_{9}{ }^{2}+\dot{z}_{9}{ }^{2}\right),
$$

l'énergie potentielle $U$ correspond aux forces centrales qui lient les particules $\alpha$ et $\beta$ et n'est fonction que de la distance des deux particules. La fonction $R$ est la somme $T+U$, qui n'est pas influencée par les forces exercées par le champ magnétique. On a donc $\frac{\mathrm{d} R^{(*)}}{\mathrm{d} t}=0$, excepté au moment d'un choc et $\boldsymbol{R}^{(1)}+\boldsymbol{R}^{(2)}=\boldsymbol{R}^{(1)^{\prime}}+\boldsymbol{R}^{(2)^{\prime}}$ au moment du choc des molécules (1) et (2). Nous traiterons deux cas de collisions élastiques : la première hypothèse sur le choc sera que chaque particule $\alpha$ ou $\beta$ d'une molécule peut se choqłer avec chaque particule d'une seconde molécule, tandis que la deuxième hypothèse sera que seulement les particules $\alpha$ se choqueront tandis que les particules $\beta$ ne subissent pas d'influence des collisions. Il faut chercher les équations accessoires aux conditions (1) et (2) pour parvenir à la solution de celles-ci. On trouve la solution $f=A \mathrm{e}^{-h R}$ pour les deux cas différents, si le gaz n'est pas en mouvement. La fonction $f$ étant entièrement indépendante du champ magnétique, le moment magnétique est nul.

\section{VIII. - Effet magnétique des électrons libres dans les métaux.}

Pour étudier l'effet magnétique des électrons libres dans l'unité de volume d'un métal, il faut calculer la valeur moyenne dans le temps de $M=\sum \frac{e}{2 c}(y \dot{z}-z \ddot{y})$, où la somme s'étend à tous les électrons contenus 
dans ce volume, $e$ représentant la charge d'un d'entre eux el $x, y, z$ ses coordonnées rectangulaires. Dans l'état stationnaire la valeur moyenne et la valeur à chaque instant doivent ètre les mèmes. Pour effectuer cette somme il nous faul la fonction distributrice. Pour ce cas, nous prenons comme fonction de Lagrange, comme dans le cas précédent, l'énergie cinétique $T$, de sorte que les moments ne diffèrent des composantes de vitesse que par un lacteur constant. La fonction de Routh coïncide avec $T$. Si on suppose que les électrons sphériques se choquent élastiquement avec les molécules (supposées en repos à cause de leur masse relativement grande) on trouve immédiatement comme solution des équations (1) et (2); $p=\varphi\left(\dot{x}^{2}+\dot{y}^{2}+\dot{z}^{2}\right)$. Le champ magnétique n'y joue aucun ròle, le moment résultant est donc nul.

Si on suppose, au contraire, que les électrons ne se choquent que mutuellement, un état peut se maintenir, où tout le système d'électrons tourne avec une vitesse angulaire arbitraire $\omega$ autour de l'axe $O X$. Si $\omega$ n'est pas nul, le moment résultant ne sera pas nul non plus, mais ce moment correspond au courant d'induction, si le système n'avait point de rotation (donc pas de moment magnétique) au moment où le champ magnétique a été établi.

\section{IX. - Remarques sur l'effet magnétique de molécules arbitraires.}

Les démonstrations données pour l'existence ou l'absence d'un moment résultant dans les divers cas traités sont tellement adaptées aux cas spéciaux, qu'on n'y reconnaìt pas aisément 'un caractère général. Pourtant on peut dire quelque chose sur le moment magnétique d'un système de molécules de constitution arbitraire. Supposons l'état de la $x^{\text {me }}$ des molécules fixé par les $n$ coordonnées $q_{1}^{(x)}, \ldots q_{v}{ }^{(x)}, \ldots q_{n}{ }^{(x)}$ et les $\dot{q}_{1}{ }^{(x)}, \ldots \dot{q}_{n}{ }^{(x}$ correspondantes. Soit $L$ la fonction complète de Lagrange, c'est-à-dire la somme des différences des énergies cinétique et potentielle et des énergies magnétique et électrique, de sorte que les équations du mouvement ont pour toutes les coordonnées d'une molécule la forme

$$
\frac{\mathrm{d}}{\mathrm{d} t}\left(\frac{\partial L}{\partial \dot{q}_{v}}\right)-\frac{\partial L}{\partial q_{v}}=Q_{v}^{\prime} .
$$

où les $Q^{\prime}$ v ne sont que les forces de collision.

Nous introduisons des moments $p_{\mathrm{v}}$ au lieu des composantes de vitesse $\dot{q}$, et une fonction $R$, comme auparavant, de sorte que les équations de mouvement ont pour loutes les coordonnées de la molécule la forme canonique 


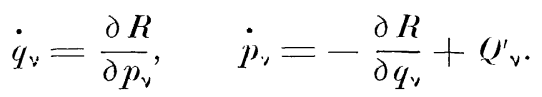

Supposons le champ magnétique produit par un courant arec lïntensité constante $\dot{q}_{0}$, où $\%_{0}$ est une coordonnée cyclique pour le système extérieur. On déduit alors immédiatement que $\frac{\mathrm{d} R^{(x)}}{\mathrm{d} t}=0$ pendant le temps que la molécule ( $)$ ne se choque pas et que $\boldsymbol{R}^{(\boldsymbol{x})}+\boldsymbol{R}^{\prime)}$ est constant pendant la collision des molécules ( $x$ ) et ( $\lambda_{\text {. }}$.

Nous nommons une grandeur $I$ une intégrale additive du mouvement, si elle a les propriétés suivanies communes avec $R$ :

$1^{\circ}$ D'ètre la somme d'une constante et de termes $I^{(\boldsymbol{x})}$, qui ne dépendent que des coordonnées et moments d'une molécule ;

$2^{\circ}$ De sorte que $I^{(x)}$ reste constant, tant que la ${ }^{\text {me }}$ molécule ne se choque pas;

$3^{\circ}$ Et que $I^{(x)}+I^{(\hat{\imath})}$ reste constant pendant une collision de la $\%^{\text {me }}$ molécule avec la $\lambda^{\text {ine }}$.

Si nous avons $l$ intégrales de ce genre $: I_{1}, I_{2}, \ldots I_{l}$,

$$
f=a_{o} \mathbf{e}^{a_{1} I_{1}+a_{2} I_{2}+\ldots .+a_{l} I_{l}},
$$

(où les $a$ sont des constantes) est une solution des équations (1) et (2), gràce aux propriétés des $I$. Si les $I_{1}, \ldots I_{l}$ sont toutes les intégrales additives du mouvement (indépendantes les unes des autres), la solution la plus générale est trouvée ('). Mais on ne sait pas d'avance pour un système arbilraire, combien d'intégrales additives du mouvement existent et la règle suivante s'appliquera quelquefois plus facilement. Si on connail autant d'intégrales additives indépendantes du mouvement quil y a de fonctions constantes pendant le mouvement d'une molécule sans choc, ou qu'il y a de fonctions constantes au moment du choc, alors la solution trouvée pour $f$ est la solution la plus générale des équations (1) el (2) $\left({ }^{2}\right)$. Pour d'autres cas, il faut un examen spécial. Dans les cas examinés, nous avions moins d'intégrales addilives du mouvement qu'il n'y avait de fonclions constantes sans choc et au choc; il fallait examiner s'il n'était pas possible de combiner les fonctions constantes sans choc et au choc, pour en former des

( ${ }^{1}$ Car si on peut trouver pour $f$ une expression qui ne soil pas définie par $I_{1}, \ldots I_{l}$, alors $\log f$ serait une intégrale additive nouvelle du mouvement et indépendante de $I_{1}, \ldots I_{l}$, ce qui est contraire à notre supposition.

$\left.{ }^{2}\right)$ Car si ce n'était pas le cas, log $f$ donnerait encore une fonction constante pendant le mouvement sans choc et $\log f^{(1)}+\log f^{(2)}$ une fonction qui ne varierait pas au moment du choc. 
fonctions nouvelles qui soient bien des intégrales additives du mouvement. Pour les cas traités il y avait, comme intégrales additives du mourement: $\boldsymbol{R}, p_{\boldsymbol{x}}, p_{y}, p_{z}, \boldsymbol{H}\left(p_{3}, p_{l}\right), \boldsymbol{H}(p)$.

Si le résultat de cet examen spécial du caractère des fonctions constantes est que $R$ est la seule intégrale additive du mouvement, on peut démontrer que le moment magnétique résultant est nul. Car le moment magnétique d'une molécule est une fonction linéaire et homogène des composantes de vitesse $\dot{q}_{\nu}$, soit $c_{1} \dot{q}_{1}+\ldots \ldots c_{\nu} \dot{q}_{v}+\ldots \ldots c_{n} \dot{q}_{n}$. Le moment résultant est donc :

$$
M=\frac{\int\left(c_{1} \dot{q}_{1}+\ldots \dot{c}_{v} \dot{q}_{v}+\ldots \dot{c}_{n} \dot{q}_{n}\right) \mathbf{e}^{-a R} \mathrm{~d} q_{1} \ldots \ldots \mathrm{d} q_{n} \mathrm{~d} p_{1} \ldots \ldots \mathrm{d} p_{n}}{\int \mathbf{e}^{-a R} \mathrm{~d} q_{1} \ldots . \mathrm{d} q_{n} \mathrm{~d} p_{1} \ldots \ldots \mathrm{d} p_{n} .} .
$$

Si nous y substituons $\dot{q}_{\nu}=\frac{\partial \boldsymbol{R}}{\partial p_{\nu}}$ et si nous intégrons pour le $v^{\text {ième }}$ terme de lintégrale, par rapport à $p_{v}$, ce terme aura comme facteur l'intégrale :

$$
\int_{-\infty}^{+\infty} \mathrm{e}^{-a R} \frac{\partial R}{\partial p_{\nu}} \mathrm{d} p_{\nu}=\left[-\frac{1}{a} \cdot \mathbf{e}^{-a R}\right]_{-\infty}^{+\infty}=0,
$$

car si $\mathrm{e}^{-a R}$ ne s'approchait pas suffisamment de zéro pour $p_{\nu}= \pm \infty$ les intégrales ne seraient pas convergentes. Chaque terme a donc le facteur zéro, le moment résultant est donc nul.

La mème démonstration est encore valable s'il y a quelques intégrales additives du mouvement, dont une. $R$, figure comme fonction de Routh pour les coordonnées de la molécule (c'est-à-dire que les équations du mouvement ont la forme (12)). tandis que les autres ne dépendent pas des $p_{v}$, pour lesquels le $c_{v}$ n'est pas nul. Dans ce cas aussi chaque terme du numérateur a un facteur nul.

Il faut donc, afin que le moment magnétique résultant ne soit pas nul, que la fonction distributrice dépende encore d'autres fonctions que de $\boldsymbol{R}$ seule et que ces autres fonctions fassent intervenir des moments $p_{v}$, qui correspondent aux mouvements dont dépend le moment magnétique. Nous avons rencontré de telles fonctions en $H\left(p_{3}, p_{l}\right)$ et $H(p)$.

\section{$\mathrm{X}$. - Discussion des travaux publiés sur ces questions.}

Nous voulons caractériser encore en quelques mots les résultats d'autres auteurs sur ce sujet en les comparant aux résultats obtenus ici. Ge sont les résultats généraux de Langevin [13] et Kroo [12], et les résultats spéciaux valables pour une des trois sortes de molécules, déduits par Lorentz [15], Kroo [12], Holm [10] et Gans [4-9]; enfin, sur l'effet magnétique des 
électrons libres des métaux, les résultats de .. J. Thomson [21]. I'oigt [23], Lorent: [1ð, 17] et Schrödinger [19].

$1^{\circ}$ Les résultats de Langevin se basent sur l'hypothèse que toutes les molécules ont le mème moment magnétique, qui peut ètre nul ou non. A un moment nul correspond le diamagnétisme, à un moment non nul le paramagnétisme. Langevin ne mentionne pas pourquoi il ne donne qu'une valeur du moment. Il fait donc implicitement l'hypothèse qu'il y a plus d'une intégrale additive du mouvement (voyez $\$ 9$ ), de sorte que le cas où il n'y en a qu'une et que le moment résultant est nul échappe à son attention.

$2^{\circ}$ Kroo obtient le résultat que la théorie des électrons ne peut expliquer que le diamagnétisme, quelle que soit la constitution de la molécule. Il obtient ce résultat, parce qu'il ne suppose qu'une intégrale additive du mouvement. Dans ce cas nous trouvons un moment résultant nul el Kroo un moment négatif, parce qu'il se sert de l'énergie totale $\boldsymbol{E}$ au lieu de la fonction de Routh pour cette intégrale $\left({ }^{1}\right)$.

$3^{\circ}$ Lorentz a traité l'effet magnétique des molécules portant un courant dans ses leçons professées à l'Université de Leyde. Il a démontré qu'il y a deux distributions de molécules dans l'extension én phase, qui sont stationnaires quant-au mouvement sans chocs. Nous avons démontré ici que ces deux distributions, qui sont identiques à deux des nôtres, sont aussi stationnaires quant-aux chocs. Les résultats sont : diamagnétisme si toutes les molécules ont le moment zéro; paramagnétisme si toutes les molécules ont le mème moment pas trop petit; pas de magnétisme si le moment des molécules peut varier au moment des chocs.

$4^{\circ}$ On peut démontrer que la fonction distributrice dont Holm se sert, ne caractérise pas un état stationnaire. Mais les formules de Holm pour le moment magnétique résultant ont par rapport à un certain système de coordonnées en rotation la mème forme que les nòtres par rapport à un système fixe, s'il y a une intégrale additive du mouvement, de sorte que les résultats sont identiques : un moment résultant nul.

$\zeta^{\circ}$ Dans son article [8] Gans a, comme Kroo, l'énergie totale $E$ au lieu de la fonction de Routh $R$ dans la fonction distributrice. Ses résultats diffèrent de ceux de Kroo, parce qu'il remarque que des coordonnées « non statistiques » peuvent exister, c'est-à-dire des coordonnées qui ne subissent pas l'influence du mouvement thermique, donc encore d'autres intégrales additives du mouvement que $R$ seule. Gans leur donne pour chaque molécule la mème valeur différente de zéro. Gans obtient donc diamagnétisme là où nous obtenons un moment résultant nul à cause de $\boldsymbol{E}$ au

(1) Voir thèse de Leyde. 
lieu de $R$; et paramagnétisme où nous obtenons, suivant les conditions, du para ou du diamagnétisme (parce qu'il donne aux molécules un moment différent de zéro). Quant-à ses autres articles nous remarquerons seulement que la fonction distributrice ne fait pas intervenir, l’énergie magnétique de la mème manière pour des systèmes de molécules porlant des magnécules (moments magnétiques invariables) et pour des systèmes dont les molécules portent un courant électrique. L'énergie magnétique s'exprime dans le premier cas par le produit scalaire du moment magnétique el de la force magnétique $(m \boldsymbol{H})$ avec le signe négatif et par ce mème produit avec le signe positif dans le second cas. Ces deux différences se compensent si on exprime la fonction distributrice en $(m H)$. On peut donc se servir des mèmes formules finales, mais on ne peut les déduire pour les deux cas de la mème manière, ce que Gans se permet $\left(^{1}\right)$.

$6^{\circ}$ Il y a une divergence d'opinion sur la question de savoir si on peut attribuer le diamagnétisme des métaux à la circulation des électrons libres. Lorentz a démontré dans ses leçons professées à l'université de Leyde, que les électrons intérieurs, dont les projections sur un plan perpendiculaire aux lignes de force parcourent des cercles complets. correspondent à un moment négatif, mais les électrons près de la périphérie du métal correspoudent à un moment positif de la mème grandeur $\left({ }^{2}\right)$. Les autres auteurs cités n'ont pas fait attention à ce dernier moment. La fonction distributrice est la mème chez tous les auteurs. Lorentz démontrait qu'elle esl stationnaire quant-au mouvement sans chocs. Nous avons démontré qu'elle est encore stationnaire quant-aux chocs spéciaux que nous avons considérés, Schrödinger l'avait fait pour des chocs d'autre caractère.

A la fin de ce résumé de ma thèse de doctorat, je tiens à remercier MM. les professeurs $\boldsymbol{H}$. A. Lorentz et $\boldsymbol{P}$. Ehrenfest pour le vif intérèt qu'ils ont porté à mon travail et les avis précieux qu'ils m'ont donné dans l'étude de cette question. Je remercie également M. le prof. $P$. Langevin, qui a bien roulu se charger d'examiner la rédaction française.

Manuscrit reçu le $1^{\text {er }}$ janvier 1921.

\section{LISTE BIBLIOGRAPHIQUE}

1. Aupère : Les lois des phénomènes électrodynamiques, 1826.

2. Boltzuann: Vorlesungen über Gastheorie, I, II.

3. P. et T. Ehrenfest : Begriffliche Grundlagen der statistischen Aufbau der Mechanik. Encykl. d. mathem. Wissensch. IV, 32.

(1) Voir thèse de Leyde.

(2) Après que la thèse de Leyde fut publiée, nous apprìmes qu'une remarque anàlogue se trouve dans la thèse de N. BонR, Kopenhagen, 1911. 


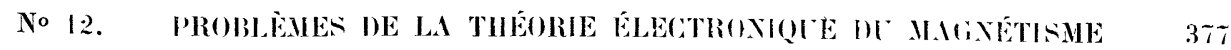

4. R. Giss : Zur Elektronentheorir des Ferromagnedismus I. Gö̈t. Narhe. Jalle. Plyss. Kl.. (1910). 1) 197.

¿. R. Gux : Zur Elektronentheorie des Foromagnelismus II. Gistt. Nache. Math. Phys. Kl.. (1911). p. 118.

6. R. Guss : Beweisen die magnelischen Ilessungen an paramagnelischen Substanzen dir Existenz einer Nullpunklsenergie? Ber. d. Deutsch. phys. Gies. (1911). p. 780.

7. R. G.xs: Der Paramagnelismus in seince Abhängigkeil ron der Temperalur und der Dichle. Ber. d. Isutsch. phys. Ges. (191't), p. 96't.

8. R. Gaxs : Slalislische Theorie des Dia-. Para- und Melamagnelismus. lun. de, Physs., 49 (1916). p. 14.9.

9. R. Guxs : Leber Paramagnelismus. Llıı. der Phys., 50 (1916). p. 163.

10. E. Hoz. : Zur slatislischen Elektronentheorio dre Magnetismus. Imm. der Phys., 44 (1911), p. 211.

11. F. Konliadesch : Lehrburh der prartischen 1'hysiti. X" Aull. 1905.

12. J.-X. Kroo : Zur stalistischen Elektronentheorio der Diëlektrizitäl und des Magnelismus. I)iss. Cristtingen 1913 ou .Lun. der Phys.. 42 (1913), p. $13 \%$ \%.

13. P. Laxgents : Magnélismo ol théorie des électrons. Inn. d. Chim. el de Phys., IIII, 5 (1908). p. 70.

14. H.-1. Lonext\% : Elektronentheorir. Encyfil. d. mathem. Wiss. Bal V. 2. Helt 1 .

1:; H.-1. Lonextz : College 1910-1911.

16. H.-A. Lonext\% : Sur la polarisation partielle de la lumière ámise par une soure lumineuse dans un champ! magnétique. Areh. V'éerl. II. $2(1899)$, p. 1.

17. H -A: Lonentz: Vortrage äber die himefische Theorie der Materie und der Elektrizitäl ron M. Planck U. 1. (1911), p. 188.

18: E Roeth : Elemenlary rigid dynamies, London. (1882).

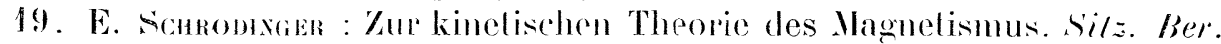
d. K. Aliad. d. Iiss. IIen, Ia. 121 (1912). p. 1303.

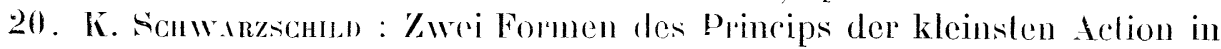
der Elektronentheorie. (i)/l. Narhs. Wath.-Phys.Kl.(1902-1903), p.12:).

21. I.-J. Thonson : Indications relatives a la constitution de la malière lournies par les rocherehes récentes sur le passage de l'électricilé à Iravers les gaz. Ropports due romgrès de physique de 1901), III-IV, Paris. p. 1 's.

22. J.-J. Thouson: The magnelic properties of systems of eorpuscles describing circular orbits. Phil. Mag.. VI. 6 (1903). p. 673.

23. W. Vorgi : Eleklronenhypothese und Theorie des Magnolismus. Imo. der Phys.. IV. 9 (1902). p. $11 \%$.

24. W. Weber : Werke III ${ }^{\text {ter }}$ Band p. 261, Ueber dio Erregung und Wirkung des Diamagnelismus, 184. 